\title{
Additional desmids (Chlorophyceae) to eastern flora of Nepal
}

\author{
Shiva Kumar Rai \\ Department of Botany \\ Post Graduate Campus, Tribhuvan University, Biratnagar, Nepal \\ E-mail: shivarai24244@yahoo.com
}

\begin{abstract}
s
Desmid of eastern Nepal has been studied. A total 15 desmids are described from different freshwater lotic and lentic habitats in the present communication. They belong to genera Closterium (8), Pleurotaenium (2), Cylindrocystis (1), Micrasterias (1), Arthrodesmus (1), Staurastrum (1) and Spondylosium (1). These are the addition of desmids from different locality to the algal flora of eastern Nepal.
\end{abstract}

Key words: Algae, Closterium, Staurastrum, Staurodesmus terai, Morang.

\section{Introduction}

Desmid flora of Nepal has been contributed by Hirano $(1955,1963,1969,1984)$ who reported 79 species of desmids from different localities in Central and Eastern Nepal. Watanabe (1971, 1982) of National Science Museum, Tsukuba has reported 24 species of Closterium from Gosain Kund, Dudh Pokhari, and Dudh Koshi river in eastern Nepal and Trisuli river, Begnas lake, and Hetauda in Central Nepal and added 5 species (Closterium ehrenbergii, Cl. jenneri, Cl. pseudopsillum, $\mathrm{Cl}$. rostratum, and $\mathrm{Cl}$. venus) and 5 varieties (Closterium closteroides var. intermedium, $\mathrm{Cl}$. dianae var. brevius, $\mathrm{Cl}$. dianae var. minus, $\mathrm{Cl}$. littorale var. crassum, and $\mathrm{Cl}$. parvulum var. maius) new to the Nepalese desmid flora. Similarly, Bando et al. (1989) have studied the desmid flora of Kathmandu and reported 49 species, 5 varieties, and 3 forms belonging to 10 genera. The frequently observed species were Closterium acerosum, $\mathrm{Cl}$. leibleinii var. recurvatum, Cosmarium javanicum, C. obtusatum, and C. subcostatum. Foerster (1965) has also reported 79 desmids from Lobuche, Pheriche, Karyolung, and Thodung areas in the Himalaya regions. Among them 72 taxa were first time reported from Nepal and 18 species were new to the world. Kusel-Fatzmann (1969) has studied both desmid and Cyanophycean algae from Kathmandu and Solukhumbu. Habib and Chaturvedi $(1995,1997)$ have reported a total 103 taxa from Mahendranagar and Mahakali river among which 63 were new for the country. Rai and Misra (2008) have reported 26 desmids (Closterium 4, Euastrum 4, Micrasterias 3, Cosmarium 7, Staurastrum 3, Onychonema 2, Hyalotheca 1, and Desmidium 2) from Koshi Tappu Wildlife Reserve, Sunsari district including 11 desmids new to Nepal and genus Onychonema first time described from the country. Similarly, Rai et al. (2008) have studied the desmids of Beez-Hazaar lake, Chitwan reporting 30 taxa belonging to 7 genera among which 11 were new to Nepal. The present paper describes the addition of some desmids reported from one or more places in eastern Nepal.

Eastern Nepal (Eastern Development Region) is situated at $26^{\circ} 20^{\prime}-28^{\circ} 08^{\prime} \mathrm{N}$ and $86^{\circ} 08^{\prime}-88^{\circ} 15^{\prime} \mathrm{E}$ in the eastern part of Nepal consists of 3 zones (Mechi, Koshi, Sagarmatha) and 16 districts occupying an area of about 28,456 sq. $\mathrm{km}$. Due to altitudinal variation, it is divisible into 5 geographical regions from south to north viz., Terai plain, Churia (Siwalik) hills, Middle mountain (Mahabharat), High mountain and Himalaya. The terai in the south has hot and humid 
sub-tropical climate, Mountains in the middle has warm and cold temperate climate and Himalaya in the north has Alpine and Tundra types of climate. The average winter temperature ranges from -30 to $18^{\circ} \mathrm{C}$ and average summer temperature from 27 to $30^{\circ} \mathrm{C}$. About $80 \%$ of the total annual rainfall occurs during monsoon in the month of June, July and August. The eastern part receives up to $3000 \mathrm{~mm}$ rainfall whereas the hill and Terai receives average 1000-2000 mm annually. It has extreme variation in elevation from $56 \mathrm{msl}$ (Kechana Kawal) to $8848 \mathrm{msl}$ (Mt. Everest, the highest peak in the world).

Eastern Nepal is rich in water resources. The major river systems in this region are Saptakoshi, Sunkoshi, Arun, Tamor, and Dudhkoshi river systems. It has many glaciers, lakes, ponds, and waterfalls especially in the mountain and Himalaya regions. Some of the important lake and ponds are Sinjema Tal, Sabha Pokhari, Gupha Pokhari, Gokyu lake, Panch Pokhari, Mai Pokhari, Rautaha Pokhari, Betana pond, Koshi Tappu wetland etc.

\section{Materials and Methods}

Algal samples were collected randomly from different lotic and lentic habitats of various geographical regions of Eastern Nepal. Epiphytic forms were collected by squeezing submerged macrophytes where as planktonic forms were collected with the help of plankton mesh (net size $10 \mu \mathrm{m})$ and preserved in $4 \%$ formalin in the plastic tubes $(250 \mathrm{ml})$. Samples were brought to the laboratory of Dept of Botany, PG Campus and temporary prepared slides were observed and microphotography under Olympus light microscope. Desmids were identified with the help of literature and monographs following Scott and Prescott (1961), Kouwets (1987), Croasdale and Flint (1986, 1988), Gerrath and John (1988), Croasdale et al. (1994), Watanabe (1995), Flint and Williamson (1998) etc.

\section{Results}

A total 15 desmids have been described under 7 genera viz., Closterium (8), Pleurotaenium (2), Cylindrocystis (1), Micrasterias (1), Arthrodesmus (1), Staurastrum (1) and Spondylosium (1). These are the reports of desmids from different locality than the previous one if any reported and addition to the desmid flora of eastern Nepal. Description of the desmids is given as follows.

1. Cylindrocystis brébissonii (Ralfs) De Bary (Fig. 11) [Basionym: Penium brebissonii Ralfs] References: Scott, A.M. and G.W. Prescott 1961, p. 8, pl. 1, fig. 3; Foerster, K. 1965, p. 29, abb. 1, figs. 1-4 (as C. Brébissonii var. jenneri); Kouwets, F.A.C. 1987, p. 196, pl. 1, figs. 1-2; Gerrath, J.F. and D.M. John 1988, p. 193, pl. 2, fig. 1; Watanabe, M.1995, p. 12, fig. 39.

Dimension: Cells $50 \mu \mathrm{m}$ long, $16.5 \mu \mathrm{m}$ broad.

Locality: Sabha Pokhari lake, 4,100 m, Sankhuwasabha district (Himalaya).

Distribution in Nepal: Khumbu, 2,000 m, Eastern Nepal (Kusel-Fetzmann, 1969); Mewa valley, Wolang Chung Gola, Eastern Nepal (Hirano, 1984); a small pond south of lake Rara, 3,030 m, Mugu (Watanabe, 1995).

2. Closterium dianae Ehr. ex Ralfs brevius (Petk.) Krieg. (Fig. 1) [Basionym: Closterium dianae f. brevior Petkoff]

References: Yacubson, S. 1980, p. 297, pl. 9, fig. 102 (as Cl. dianae); Watanabe, M. 1982, p. 50, fig. 1j; Kouwets, F.A.C.1987, p. 201, pl. 2, figs. 19-20; Bando, T. et al. 1989, p. 3, fig. 1d.

Dimension: Cells $180 \mu \mathrm{m}$ long, $22.5 \mu \mathrm{m}$ broad; apices $2.8-4 \mu \mathrm{m}$ broad, $145 \mu \mathrm{m}$ distant. 
Locality: Sarouchia pond, 72 m, Biratnagar, Morang district (Terai).

Distribution in Nepal: Paddy fields at Hetauda, 500 m, Makawanpur (Watanabe, 1982; Bando, et al. 1989); a pond near Police Station, Mahendranagar, Kanchanpur (Habib \& Chaturvedi, 1997).

3. Closterium incurvum Bréb. (Fig. 2) [Synonym: Closterium venus var. incurvum (Bréb.) Krieger]

References: Croasdale, H. and E.A. Flint 1986, p. 61, pl. 6, figs. 1-2; Kouwets, F.A.C. 1987, p. 203, pl. 2, figs. 12-13; Bando, T. et al. 1989, p. 4, figs. 2 f-g; Prasad, B.N. and P.K. Misra 1992, p. 107, pl. 16, fig. 8; Flint, E.A. and D.B. Williamson 1998, p. 77, pl. 2, fig. 6.;

Dimension: Cells $92 \mu \mathrm{m}$ long, $12 \mu \mathrm{m}$ broad; apices 2-3 $\mu \mathrm{m}$ broad, $68 \mu \mathrm{m}$ distant.

Locality: MMAM Campus pond, $72 \mathrm{~m}$, Biratnagar, Morang district (Terai).

Distribution in Nepal: Dudh Pokhari lake, 4,750 m, Gokyu, Solukhumbu, Eastern Nepal (Watanabe, 1982; Bando et al., 1989).

4. Closterium lanceolatum Kütz. ex Ralfs (Fig. 6) [Synonym: Closterium acerosum var. lanceolatum (Kützing ex Ralfs) Playfair]

References: Croasdale, H. and E.A. Flint 1986, p. 63, pl. 9, figs. 1-2.

Dimension: Cells $301 \mu \mathrm{m}$ long, $47 \mu \mathrm{m}$ broad; apices 4-5 $\mu \mathrm{m}$ broad.

Locality: Sarouchia pond, $72 \mathrm{~m}$, Biratnagar, Morang district (Terai).

Distribution in Nepal: A pool at Boudhanath, Kathmandu, 13,00 m. (Bando et al., 1989).

5. Closterium littorale Gay (Fig. 3)

References: Tiffany, L.H. and M.E. Britton 1952, p. 173, pl. 51, fig. 544; Gerrath, J.F. and D.M. John 1988, p. 200, pl. 2, fig. 15; Prasad, B.N. and P.K. Misra 1992, p. 111, pl. 17, fig. 9.

Dimension: Cells 170-174 $\mu \mathrm{m}$ long, 19-20 $\mu \mathrm{m}$ broad; apices 4-5 $\mu \mathrm{m}$ broad.

Locality: Fish pond at Everest Science Centre, 72 m, Santi Ban, Sunsari district (Terai).

Distribution in Nepal: Cl. littorale var. crassum reported from Trisuli river, $500 \mathrm{~m}$, Nuwakot (Watanabe, 1982).

6. Closterium moniliferum (Bory) Ehr. ex Ralfs var. gracile Foerster (Fig. 4)

References: Gerrath, J.F. and D.M. John 1988, p. 201, pl. 5, figs. 1-2 (as var. concavum); Bando, T. et al. 1989 , p. 7 , fig. $2 \mathrm{k}$.

Dimension: Cells 225-300 $\mu \mathrm{m}$ long, 36-39 $\mu \mathrm{m}$ broad; apices 7.5-9 $\mu \mathrm{m}$ broad, 247-276 $\mu \mathrm{m}$ distant.

Locality: Sarouchia pond, $72 \mathrm{~m}$, Biratnagar, Morang district (Terai).

Distribution in Nepal: A small pool near Swayambhu, 1,350 m, Kathmandu (Bando et al., 1989).

7. Closterium praelongum Bréb. var. brevius (Nordst.) Krieg. (Fig. 5) [Basionym: Closterium praelongum f. brevius Nordstedt; Synonym: Closterium praelongum subsp. brevius (Nordstedt) Sarim et Faridi; Closterium praelongum f. brevius Nordstedt]

References: Croasdale, H. and E.A. Flint 1986, p. 67, pl. 9, figs 4-7; Bando, T. et al. 1989, p. 7 , fig. $1 \mathrm{~h}$.

Dimension: Cells 341-370 $\mu \mathrm{m}$ long, 18-19 $\mu \mathrm{m}$ broad; apices 4-6 $\mu \mathrm{m}$ broad.

Localities: Malaya Roadside ditches, 72 m, Biratnagar, Morang district (Terai); Birat Pokhari (Pond), 135 m, Anarmani, Jhapa district (Terai).

Distribution in Nepal: Ring Road ditches at Chabahil, 1,300 m, Kathmandu (Bando et al., 1989). 
8. Closterium ralfsii Bréb. ex Bréb. var. hybridum Rabenh. (Fig. 7)

References: Scott, A.M. and G.W. Prescott 1961, p. 13, pl. 1, fig. 25; Yacubson, S. 1980, p. 298, pl. 9, fig. 108; Kouwets, F.A.C. 1987, p. 207, pl. 4, fig. 2.

Dimension: Cells $570 \mu \mathrm{m}$ long, 38-39 $\mu \mathrm{m}$ broad; apices 9-11 $\mu \mathrm{m}$ broad.

Locality: Birat Pokhari (Pond), 135 m, Anarmani, Jhapa district (Terai).

Distribution in Nepal: A pond near Mahendranagar road, Kanchanpur (Habib \& Chaturvedi, 1997).

9. Closterium tumidum Johns. (Fig. 8) [Basionym: Closterium cornu Ehr. ex Ralfs]

References: Scott, A.M. and G.W. Prescott 1961, p. 41, pl. 1, fig. 16; Yacubson, S. 1980, p. 298, pl. 9, fig. 107; Watanabe, M. 1982, p. 58, fig. 6g; Gerrath, J.F. and D.M. John 1988, p. 205, pl. 5, figs. 6-7; Bando, T. et al. 1989, p. 8, fig. 1e (as Cl. tumidum var. tumidum); Prasad, B.N. and P.K. Misra 1992, p. 119, pl. 17, fig. 11.

Dimension: Cells $107.5 \mu \mathrm{m}$ long, $17.5 \mu \mathrm{m}$ broad; apices 5-7.5 $\mu \mathrm{m}$ broad.

Locality: Fish pond at Everest Science Centre, 72 m, Santi Ban, Sunsari district (Terai).

Distribution in Nepal: A shallow pool above Gosainkunda, 4,500 m, Rasua and rice fields at Hetauda, 500 m, Makawanpur (Watanabe, 1982); Mahendranagar, Kanchanpur (Habib \& Chaturvedi, 1997).

10. Pleurotaenium baculoides (Roy et Biss.) Playf. (Fig. 9) [Basionym: Docidium baculoides Roy et Bisset; Synonym: Docidium trabecula var. baculoides (Roy et Bissett) Playfair]

References: Scott, A.M. and G.W. Prescott 1961, p. 14, pl. 3, fig. 5; Nurul Islam, A.K.M. 1970, p. 912, pl. 4, figs. 8-9; Kouwets, F.A.C. 1987, p. 207, pl. 6, fig. 8; Bando, T. et al. 1989, p. 11, fig. $3 \mathrm{~g}$.

Dimension: Cells $387 \mu \mathrm{m}$ long, $30 \mu \mathrm{m}$ broad; isthmus $20 \mu \mathrm{m}$ wide; apices $20-21 \mu \mathrm{m}$ broad.

Remarks: Present specimen has shorter but slightly broader dimension with wider isthmus.

Locality: Sarouchia pond, 72 m, Biratnagar, Morang district (Terai).

Distribution in Nepal: Taudaha, 1,350 m, Kathmandu (Bando et al., 1989); Bees hazar lake, 286 m, Tikauli, Chitwan (Rai et al., 2008).

11. Pleurotaenium trabecula (Ehr.) ex Naeg. (Fig. 10)

References: Scott, A.M. and G.W. Prescott 1961, p. 18, pl. 3, fig. 4; Nurul Islam, A.K.M. and A.K. Yusuf Haroon 1980, p. 564, pl. 4, fig. 56; Kouwets, F.A.C. 1987, p. 208, pl. 6, fig. 7.

Dimension: Cells $568 \mu \mathrm{m}$ long, 39-40 $\mu \mathrm{m}$ broad; isthmus 32-35 $\mu \mathrm{m}$ wide; apices 20-22 $\mu \mathrm{m}$ broad.

Locality: Malaya Roadside ditches, 72 m, Biratnagar, Morang district (Terai).

Distribution in Nepal: Pond at Ankhu Khola, 640 m, Luitel Bhanjyang, 770 m, Gorkha and Pisang, 3,100 m, Manang (Hirano, 1955).

12. Micrasterias mahabuleshwarensis Hobs. var. surculifera Legerh. (Fig. 12)

References: Scott, A.M. and G.W. Prescott 1961, p. 50, pl. 16, figs. 1-2; Nurul Islam, A.K.M. 1970, p. 920, pl. 7, figs. 7-8; pl. 11, fig. 5; pl. 9, fig. 3 (as M. mahabuleshwarensis morpha); Nurul Islam, A.K.M. and A.K. Yusoof Haroon 1980, p. 572, pl. 3, fig. 51; Bando, T. et al. 1989, p. 15, fig. 6a (as M. mahabuleshwarensis).

Dimension: Cells $110 \mu \mathrm{m}$ (without processes)-145 $\mu \mathrm{m}$ (with processes) long, $125 \mu \mathrm{m}$ broad; isthmus $22.5 \mu \mathrm{m}$ wide; polar lobes $24 \mu \mathrm{m}$ (without processes)-68 $\mu \mathrm{m}$ (with processes) broad.

Locality: Routaha Pokhari (Lake), 1,770 m, Udayapur district (Mountain).

Distribution in Nepal: Bees hazar lake, 286 m, Tikauli, Chitwan (Rai et al., 2008). 

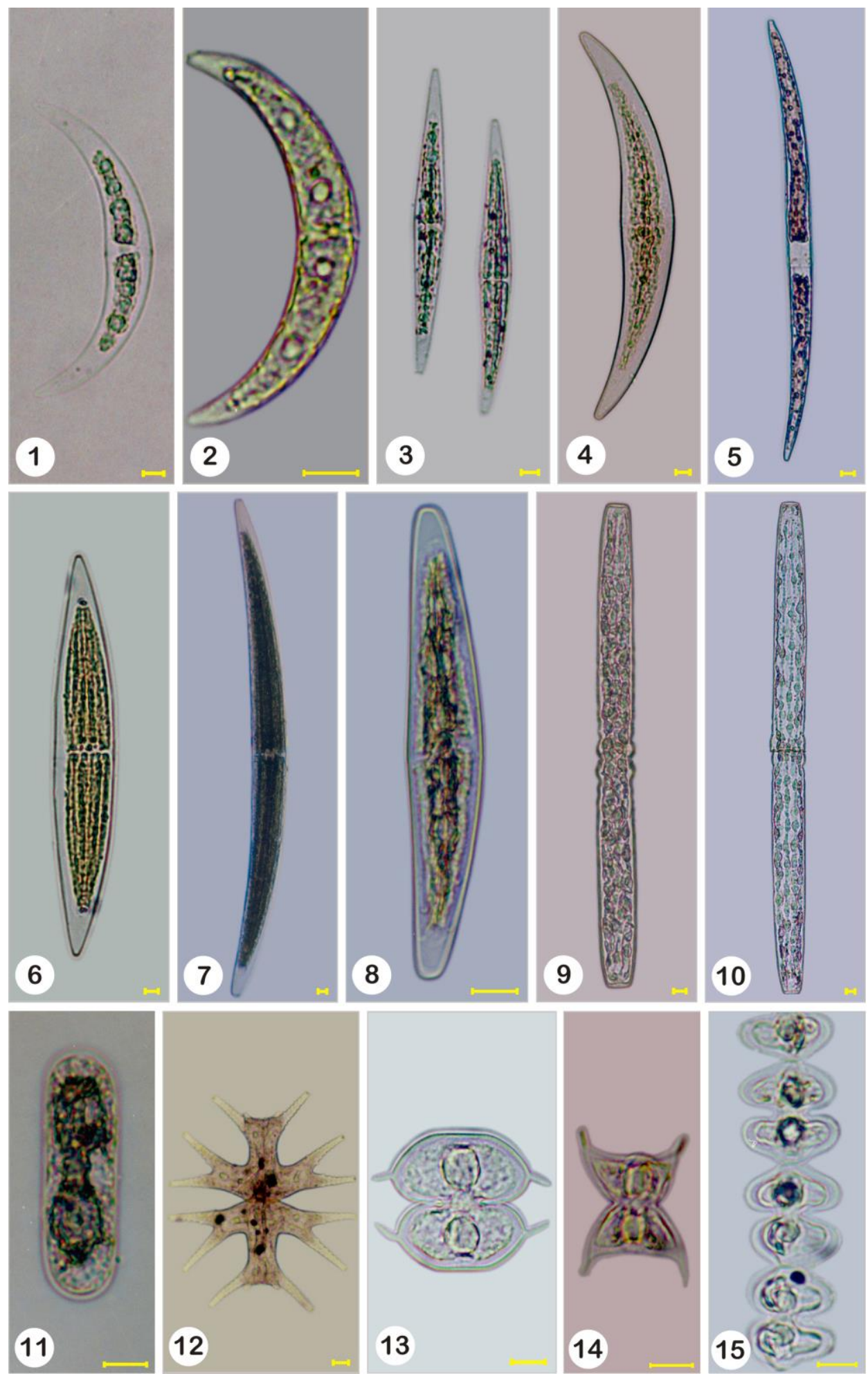
Figures 1. Closterium dianae brevius 2. Closterium incurvum 3. Closterium littorale 4. Closterium moniliferum var. gracile 5. Closterium praelongum var. brevius 6. Closterium lanceolatum 7. Closterium ralfsii var. hybridum 8. Closterium tumidum 9. Pleurotaenium baculoides 10. Pleurotaenium trabecula 11. Cylindrocystis brébissonii 12. Micrasterias mahabuleshwarensis var. surculifera 13. Staurodesmus convergens var. ralfsii 14. Staurodesmus dejectus var. dejectus 15. Spondylosium nitens var. triangulare f. javanicum (scales measure $10 \mu \mathrm{m}$ )

13. Staurodesmus convergens (Ehr.) Teil. var. ralfsii Teil. (Fig. 13) [Basionym: Arthrodesmus convergens Ehr. ex Ralfs]

References: Croasdale et al. 1994, p. 42, pl. 75, figs 13-14.

Dimension: Cells $42 \mu \mathrm{m}$ long, 39.5-42 $\mu \mathrm{m}$ broad; isthmus $12.5 \mu \mathrm{m}$ wide; spines $6.2-7 \mu \mathrm{m}$ long.

Locality: Fish pond at Everest Science Centre, 72 m, Santi Ban, Sunsari district (Terai).

Distribution in Nepal: Bees hazar lake, 286 m, Tikauli, Chitwan (Rai et al., 2008 as Arthrodesmus convergens var. curtus).

14. Staurodesmus dejectus (Bréb. ex Ralfs) Teil. var. dejectus (Fig. 14)

References: Croasdale et al. 1994, p. 45, pl. 66, figs. 3-9.

Dimension: Cells 22.5-23.7 $\mu \mathrm{m}$ (without spines) to 34-35 $\mu \mathrm{m}$ (with spines) long, $22.5 \mu \mathrm{m}$ (without spines) to $25 \mu \mathrm{m}$ (with spines) broad; isthmus $10 \mu \mathrm{m}$ wide; spines 3.8-4.7 $\mu \mathrm{m}$ long.

Locality: Fish pond at Everest Science Centre, 72 m, Santi Ban, Sunsari district (Terai).

Distribution in Nepal: As Staurastrum dejectum Bréb. ex Ralfs - Khumbu, 5,000 m, Eastern Nepal (Kusel-Fetzmann, 1969); a small pond south of Rara lake, 3,030 m, Mugu (Watanabe, 1995).

15. Spondylosium nitens (Wall.) Arch. var. triangulare Turn. f. javanicum Gutw. (Fig. 15)

References: Scott, A.M. and G.W. Prescott 1961, p. 121, pl. 60, fig. 10; Nurul Islam, A.K.M. 1970, p. 932, pl. 3, figs. 7, 11; Prasad, B.N. and P.K. Misra 1992, p. 202, pl. 26, fig. 13.

Dimension: Cells $27.5 \mu \mathrm{m}$ long, $25 \mu \mathrm{m}$ broad; isthmus $6.3 \mu \mathrm{m}$ wide.

Locality: Fish pond at Everest Science Centre, 72 m, Santi Ban, Sunsari district (Terai).

Distribution in Nepal: The lakes Phewa, Rupa, Begnas, and Khaste, 960 m, Kaski (Hickel, 1973); a small pond near Mahendranagar Sabzi Market, Kanchanpur (Habib \& Chaturvedi, 1995).

\section{References}

Bando, T., T. Nakano \& M. Watanabe. 1989. The desmid flora of Kathmandu, Nepal. Bull. Natn. Sci. Mus., Ser. B, Tokyo. 15: 1-25.

Croasdale, H. \& E.A. Flint. 1986. Flora of New Zealand. Freshwater algae, chlorophyta, Desmids: with ecological comments on their habitats. Vol. I., Government Printer, Wellington, New Zealand.

Croasdale, H. \& E.A. Flint. 1988. Flora of New Zealand. Freshwater algae, chlorophyta, Desmids: with ecological comments on their habitats. Vol. II., Botany Division, D.S.I.R., Christchurch, New Zealand.

Croasdale, H., E.A. Flint \& M.M. Racine. 1994. Flora of New Zealand. Freshwater algae, chlorophyta, Desmids: with ecological comments on their habitats. Vol. III., Manaakiwhenua Press, Lincoln, New Zealand.

Flint, E.A. \& D.B. Williamson. 1998. Desmids (Chlorophyta) in two ponds in Central Canterbury, New Zealand. Algological Studies 91: 71-100. 
Foerster, K. 1965. Beitrag zur kenntais der Desmidiaceen-flora von Nepal. Erg. Forsch-unturn Nepal Himalaya. Khumbu Himal 1, 2: 25-58. http://dx.doi.org/10.1007/978-3-642-92901-4_1

Gerrath, J.F. \& D.M. John. 1988. The desmids of Ghana, West Africa- I. Nova Hedwigia 46(1-2): 187-230.

Habib, I. \& U.K. Chaturvedi. 1995. On some desmids from Nepal. J. Ind. bot. Soc. 74(1-4): 277-282.

Habib, I. \& U.K. Chaturvedi. 1997. Contribution to the knowledge of desmids from Nepal. Phykos 36(1-2): 27-36.

Hickel, B. 1973. Limnological investigations in lakes of Pokhara valley, Nepal. Int. Rev. ges Hydrobiol. 58(5): 659-672. http://dx.doi.org/10.1002/iroh.19730580506

Hirano, M. 1955. Fresh water algae. In: Fauna and flora of Nepal Himalaya, (Kihara, H. Ed.), Fauna and Flora Research Society, Kyoto University, Kyoto, Japan. pp. 5-42.

Hirano, M. 1963. Fresh water algae from the Nepal Himalaya, collected by a member of the Japanese Climbing Expedition. Contr. Biol. Lab., Kyoto Univ., Japan. 16: 1-23.

Hirano, M. 1969. Fresh water algae from Langtang Himal, Nepal Himalaya. Contr. Biol. Lab., Kyoto Univ., Japan. 22: 1-42.

Hirano, M. 1984. Fresh water algae from East Nepal. Study reported of Baika Junior College 32: 197-215.

Kouwets, F.A.C. 1987. Desmids from the Auvergne (France). Hydrobiol. 146: 193-263. http://dx.doi.org/10.1007/BF00016347

Kusel-fetzmann, E. 1969. Einige Algen aus Nepal. Khumbu Himal (Berlin) 1(6): 37-56.

Nurul Islam, A.K.M. \& A.K. Yusuf Haroon. 1980. Desmids of Bangladesh. Int. Revue ges. Hydrobiol. 65(4): 551-604.

Nurul Islam, A.K.M. 1970. Contributions to the knowledge of desmids of East Pakistan, Part I. Nova Hedwigia 20: 903-983.

Prasad, B.N. and P.K. Misra. 1992. Fresh water algal flora of Andaman and Nicobar islands, Vol. II, Singh, B. \& M.P. Singh Publ., Dehradun, India. 284p.

Rai, S.K. and P.K. Misra. 2008. On some desmids from Koshi Tappu Wildlife Reserve, Nepal. Ecoprint 15: 47-58.

Rai, S.K., R.K. Rai \& N. Paudel. 2008. Desmids from Bees-hazaar lake, Chitwan, Nepal. Our Nature 6: 58-66.

Scott, A.M. \& G.W. Prescott. 1961. Indonesian desmids. Hydrobiologia 17(1-2):1-132. http://dx.doi.org/10.1007/BF00040416

Tiffany, L.H. \& M.E. Britton. 1952. The algae of Illinois. Hafner publishing Co., New York. 407p.

Watanabe, M. 1971. Algae in Himalaya no doshokubutsu (in Japanese). The Hokkaido Univ. Sci. Expedition to Nepal Himalaya 1968. pp. 93-104.

Watanabe, M. 1982. Observations on the genus Closterium from Nepal. In: Reports on the Cryptogamic study in Nepal (Otani, Y. Compiled). National Science Museum, Tokyo, Japan. pp. 47-59.

Watanabe, M. 1995. Algae from Lake Rara and its vicinities, Nepal Himalayas. In: Cryptogams of the Himalayas, Vol. 3, Nepal and Pakistan, (Watanabe, M. \& H. Hagiwara Eds.), National Science Museum, Tsukuba, Japan. pp. 1-17.

Yacubson, S. 1980. The phytoplankton of some fresh water bodies from Zulia State (Venezuela). Nova Hedwigia 33: 279-339. 